\title{
Sudden, rapid acquisition of conditioned suppression (CER)
}

\author{
T. GRAY \\ Concordia University, Sir George Williams Campus, Montreal, Quebec H3G IM8, Canada
}

\begin{abstract}
Trial-by-trial acquisition of the CER was measured in a number of groups with different CSs. Analysis of the acquisition curves for individual animals revealed that, for the majority of animals, CS-US associations developed in a stepwise rather than a gradual fashion. In some cases, with the most effective CS, conditioning occurred with one CS-US pairing.
\end{abstract}

Although the results from CER studies have been widely cited as empirical support for theoretical accounts of conditioning (Mackintosh, 1974; Rescorla \& Wagner, 1972), little explicit attention has been paid to the nature of the acquisition function of the CER itself. Trial-by-trial acquisition curves have not been typically presented. The CER technique used in many studies presents four CS-US pairings during a daily barpressing session. Suppression ratios are then calculated over blocks of four trials, thus giving daily suppression ratios rather than trial-by-trial ratios. There is usually a large increase in suppression evident between the Day 1 and Day 2 ratios, which gives the impression that a sudden acquisition has taken place (Kamin \& Schaub, 1963). The decrease in suppression ratios is largely an artifact of the averaging procedure, in that the first daily ratio includes Trial 1, on which no suppression occurs, and the second daily ratio includes trials on which virtually complete suppression has been attained. In the cases where trialby-trial acquisition data have been presented (e.g., Kamin, 1969), a large drop in suppression ratios between Trial 4 (last trial on Day 1) and Trial 5 (first trial on Day 2) is still often observed. However, a gradual acquisition curve can also be observed in some cases, particularly when mean, rather than median, suppression ratios are presented. Whether the acquisition curve appears gradual or sudden also depends on the effectiveness of the CS and US parameters.

It should also be clear that averaging over a number of animals, even when trial-by-trial ratios are presented, could still mask the basic form of the acquisition curve; for example, some animals may condition precipitously between Trials 2 and 3, others between Trials 3 and 4, etc. The data presented here will show that, for the majority of animals, a sudden, rather than a gradual, acquisition curve is normal.

This research was supported by a grant from the National Research Council of Canada (A9789). Reprint requests should be sent to T. Gray, Department of Psychology, Concordia University, Sir George Williams Campus, 1455 de Maisonneuve Boulevard, Montreal, Quebec H3G 1M8, Canada.

\section{METHOD}

\begin{abstract}
Subjects and Apparatus
The subjects were experimentally naive, male hooded rats that weighed between 250 and $275 \mathrm{~g}$ when they were received from the supplier. The apparatus consisted of eight Grason-Stadler conditioning units individually housed in sound-attenuating boxes. The appropriate timers, relays, and counters necessary for automatic control of the units were located in an adjacent room.
\end{abstract}

\section{Procedure}

Initial training was identical for all animals. Over a period of 10 days, all animals were food deprived to $75 \%$ of their ad-lib weights, and they were maintained on a once-a-day feeding schedule. The initial magazine training was followed by three, daily, 2-h barpress sessions for food on a 2.5-min VI schedule. Reinforcements were $.045-\mathrm{g}$ Noyes food pellets. Pretest presentations of the CS to be used later in conditioning were given during the next 3 days. The specific pretest experience given differed depending on the animals' group (see below). A delay procedure, with CS durations of 3 min was always used. Noise CSs were always $80-\mathrm{dB}$ white noise generated by a Grason-Stadier $901 \mathrm{~B}$ noise generator, and light CSs always consisted of the illumination of the conditioning unit with a $10-\mathrm{W}$ bulb. Animals that were to receive a light $\mathrm{CS}$ were always trained in complete darkness. The US, a .5-sec, 1.0-mA shock presented through the grid floor of the conditioning unit, was produced by Grason-Stadler Model E1064GS shock generators. Except where otherwise stated, all animals received four CS-US pairings superimposed on the regular, daily, 2 -h barpress session. The CS was programmed to occur 18 , 38,64 , and $88 \mathrm{~min}$ after the start of the 2 -h session.

\section{Dependent Measure}

The measure reported is a suppression ratio that compares the animal's rate of responding during the $\mathrm{CS}$ to the rate during an immediately preceding period of equal duration. The ratio is $B /(A+B)$, where $B$ represents the number of responses during the $\mathrm{CS}$ and $\mathrm{A}$ the number in the immediately preceding 3-min period. A ratio of .00 indicates complete suppression of barpressing during the $\mathrm{CS}$, while a ratio of .50 indicates no change in the rate of barpressing.

\section{Different CS Conditions}

Data from a number of different subgroups that were part of previous and ongoing experiments were combined to form three main groups that differed only in the type of CS used:

Group A: 80-dB white noise CS (80-dB)-The first group, which was conditioned with a $80-\mathrm{dB}$ white noise $\mathrm{CS}$, consisted of 32 animals ( 4 subgroups of 8 animals each). The pretest experience for this group consisted of four CS presentations. These animals received at least 4 days of conditioning, i.e., 16 trials.

Group B: Compound noise-plus-light $\mathrm{CS}(\mathrm{L}+\mathrm{N})-\mathrm{This}$ group, 
which consisted of 32 animals, 8 per subgroup, was conditioned with a simultaneous compound of light and noise. Pretest experience for these animals consisted of four presentations of the light, four of the noise, and four of the compound over the course of 3 pretest days. These animals all received at least 12 conditioning trials.

Group C: Pulsing light and noise (pulses)-The CS for these 24 animals, 8 per subgroup, was a series of 1 -sec-duration alternating light and noise pulses. The pretest experience for this group consisted of four presentations of the pulsed light and four presentations of the pulsed noise over two daily pretest sessions. These animals all received at least 12 conditioning trials.

As previously stated, all of the above rats were conditioned with the traditional four CS-US pairings per day. Another group of animals (Group D) was conditioned in identical fashion to Group A animals (80-dB) except that they received five trials on the first conditioning day. The fifth trial occurred $20 \mathrm{~min}$ after the regularly scheduled fourth trial.

\section{Classification of Animals}

All animals were classified into one of three categories, namely, sudden acquisition, gradual acquisition, and nonacquisition. An animal qualified for the sudden acquisition category only if a drop in suppression ratio of .25 or more occurred between two successive trials. For example, trial-by-trial ratios for an animal might be $.52, .55, .41, .08, .03$. This animal qualifies as "sudden" in that a drop of .33 occurred between Trial 3 and Trial 4. An animal was classified as "gradual" if no drop of .25 or more occurred between any two successive trials, for example, .46, $.51, .47, .26, .15, .00$. An animal that did not show suppression ratios below .25 within 12 trials of conditioning was classified as "nonacquisition."

\section{RESULTS AND DISCUSSION}

Table 1 shows the results of the classification procedure for the 96 animals of Groups A, B, C, and D. The number of animals showing a sudden drop in suppression ratio on a particular trial is indicated along with the number of gradual and nonacquisition animals. It is clear that the majority of animals, $77 \%$, showed sudden acquisition. It is also clear that the sudden drop does not always occur between Trials 4 and 5. The 24-h period between Trials 4 and 5 does not seem to have any special significance in that $50 \%$ of the animals show the large drop on Trial 4 or earlier. The results from Group $D$, which received five rather than four trials on the 1st conditioning day, also indicate that the 24 -h period is not crucial. Median suppression ratios for the first five trials for Group D were $.58, .53, .49, .45$, and .04 , respectively.

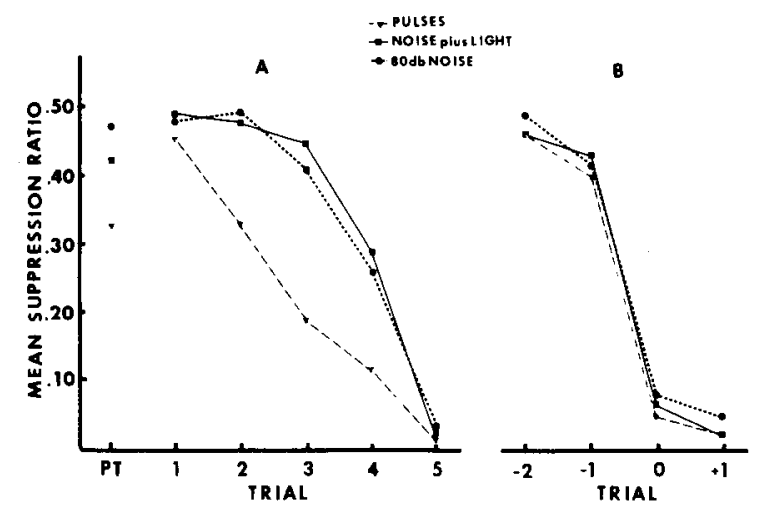

Figure 1. Trial-by-trial acquisition of conditioned suppression. Panel A shows the usual acquisition curve derived from averaging suppression ratios for each conditioning trial. Panel B shows the acquisition curve that is derived when the ratios are averaged taking into account the trial on which the large change in suppression occurred.

Figure 1 shows trial-by-trial acquisition of the conditioned suppression for all animals. Panel A presents mean suppression ratios for the animals that were classified as "sudden." Also shown are the suppression ratios for the first pretest (CS without shock) presentation. The relatively large amount of suppression to the pulsed CS habituates within the four pretest trials, as can be seen from the suppression ratio on the first conditioning trial. Panel B also shows the mean suppression ratios for the sudden animals, but in this case the trial on which the sudden drop occurred is taken into account. Trial 0 is the trial on which the drop appeared, Trials -1 and -2 are the two preceding trials. Trial +1 is the trial after the trial on which the drop occurred. It is clear that essentially complete suppression has occurred within five conditioning trials. The "gradual" acquisition of suppression is clearly evident in Panel A, with acquisition to the more effective pulsing CS greatly accelerated in comparison to the other CS categories. Panel B, however, demonstrates in dramatic fashion that the acquisition curve for individual animals is characterized by a stepwise function.

The slight increase in suppression from Trial -2 to Trial -1 was significant for the combined $80-\mathrm{dB}$ and $\mathrm{L}+\mathrm{N}$ groups $[\mathrm{t}(47)=3.95, \mathrm{p}<.001]$. Data from the "pulses" groups are not included in this

Table 1

Classfication of Animals Indicating the Number of Animals Showing Sudden Acquisition on a Particular Trial

\begin{tabular}{|c|c|c|c|c|c|c|c|c|c|}
\hline \multirow[b]{2}{*}{ Group } & \multicolumn{6}{|c|}{ Trial on Which Drop Occurred } & \multicolumn{3}{|c|}{ Classification } \\
\hline & $\mathbf{N}$ & 2 & 3 & 4 & 5 & 6 & Sudden & Gradual & NA* \\
\hline$A(80-d b)$ & 32 & & 5 & 7 & 14 & & 26 & 4 & 2 \\
\hline$B(L+N)$ & 32 & & & 9 & 13 & & 22 & 5 & 5 \\
\hline C (Pulses) & 24 & 6 & 5 & 3 & 4 & 1 & 19 & 5 & \\
\hline D (5 Trial) & 8 & & & 2 & 3 & 2 & 7 & 1 & \\
\hline Total & 96 & 6 & 10 & 21 & 34 & 3 & 74 & 15 & 7 \\
\hline Percent & & 8 & 14 & 28 & 46 & 4 & 77 & 16 & 7 \\
\hline
\end{tabular}

*Nonacquisition. 
comparison because many of these animals showed the sudden drop between Conditioning Trials 1 and 2; there was, therefore, no trial that could be designated -2 for these animals. The differences between the -1 trial suppression and the initial pretest suppression for the combined $80-\mathrm{dB}$ and $\mathrm{L}+\mathrm{N}$ groups was not significant $[\mathrm{t}(47)=1.58, \mathrm{p}>.10]$. The amount of suppression evident on the trial before the drop trial was, therefore, no greater than on the first pretest (unconditioned) presentation of the CS.

The mean change in suppression ratio from Trial -1 to Trial 0 was $.36, .34$, and .35 for the $L+N, 80-d B$, and pulses groups, respectively. These actual mean changes, it will be noted, are considerably in excess of the .25 change that was employed as a criterion for classifying an animal as showing sudden acquisition.

The choice of a change in suppression ratio of .25 as the criterion for sudden acquisition might be questioned. A decision had to be made as to what constituted a large vs. a small change in suppression ratio. The .25 change was chosen in that it was nominally half of the total change that could occur, assuming that the suppression on Conditioning Trial 1 was .50 on the average. As noted, the actual amount of change that occurred for animals classified as sudden, on the basis of the .25 criterion, was .35 . We are not dealing, then, with an actual change of debatable magnitude. An average change of .35 for the three groups is very large. The three curves plotted in Panel B of Figure 1 are virtually superimposed even though the data were gathered from different experiments with different CS characteristics. The three groups thus could be viewed as constituting an impressive cross validation of the criterion used for classifying the animals. The additional three animals in Group D constitute a further, separate, validation.

It might also be objected that the .25 change criterion could be met by an animal dropping from a ratio of .55 to a ratio of .30 , and that a ratio of .30

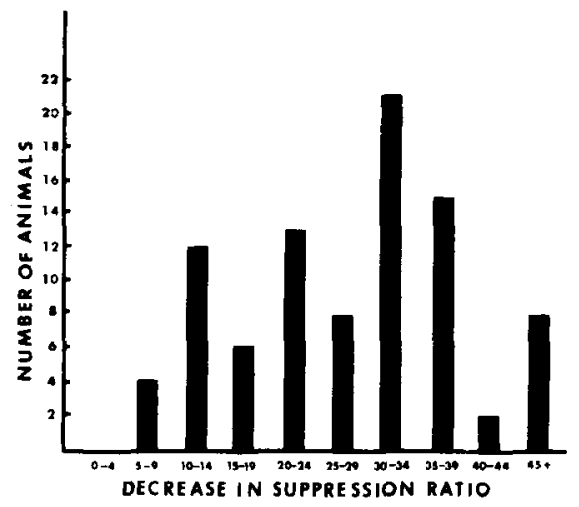

Figure 2. Number of animals showing a drop in suppression ratio of a particular magnitude. The drop was between the trial on which the suppression ratio reached .10 or below and the preceding trial. Decimal points have been left out.
Table 2

Mean Pre-CS (Baseline) Response Rates for Trial 0 Trial -1 , and Trial +1

\begin{tabular}{cccc}
\hline & \multicolumn{3}{c}{ Trial } \\
\cline { 2 - 4 } Group & -1 & 0 & +1 \\
\hline A (80-db) & 55 & 59 & 54 \\
B (L+N) & 50 & 51 & 40 \\
C (Pulses) & 47 & 52 & 50 \\
\hline
\end{tabular}

reflects, at best, only moderate suppression. Perhaps a more stringent and conservative way to show the sudden nature of the acquisition would be to select a criterion representing substantial conditioned suppression and to look at how much of a change there was between that trial and the previous trial for all animals without classifying them as sudden or gradual.

Figure 2, therefore, shows the number of animals showing a particular amount of change between the trial on which they attained a suppression ratio of .10 or less and the preceding trial. Only the animals classified as nonconditioned were excluded in the preparation of this frequency histogram. Even though the histogram underestimates the number of animals showing large changes between a pair of trials because of the additional requirement that the ratio of the second trial of the pair be .10 or below, $52 \%$ of the changes were equal to or greater than .30 and $61 \%$ were equal to or greater than the .25 criterion of change.

Table 2 presents the mean pre-CS (baseline) response rates for the trial on which the drop occurred, and the preceding and subsequent trials. It is clear that the sudden changes in suppression ratios were not dependent on sudden changes in baseline responses that might be indicative of any sudden nonassociative change in the animal's emotional, affective, or hormonal state.

The results presented above indicate that, with the CS and US parameters employed, the acquisition of the CER is very rapid; that is, it takes few trials, and for the majority of animals it appears to be acquired suddenly it may be noted that an implicit assumption here is that the sudden drop in suppression ratios reflects the fact that the conditioned association has been completely established. It must be admitted, however, that were it possible to measure suppression ratios below .00 , further learning and perhaps further large changes could be observed. Resistance to extinction tests after various amounts of training beyond the trial of the sudden drop would be of relevance here.

Although data from such studies have been used as empirical support for Rescorla and Wagner's (1972) popular theory, the rapidity and suddenness has not been taken into account. Very recently, however, Frey and Sears (1978) have presented a version of the 
Rescorla-Wagner model that incorporates a catastrophe rule that helps account for sudden changes in behavior such as have been described above.

\section{REFERENCES}

Frey, P. W., \& Sears, R. J. Model of conditioning incorporating the Rescorla-Wagner associative axiom, a dynamic attention process, and a catastrophe rule. Psychological Review, $1978,85,321-340$.

KAMIN, L. J. Predictability, surprise, attention, and conditioning. In B. A. Campbell \& R. M. Church (Eds.), Punishment and aversive behavior. New York: Appleton-Century-Crofts, 1969.
Kamin, L. J., \& Schaub, R. E. Effects of conditioned stimulus intensity on the conditioned emotional response. Journal of Comparative and Physiological Psychology, 1963, 56, 502-507.

Mackintosh, N. J. The psychology of animal learning. London: Academic Press, 1974.

Rescorla, R. A., \& Wagner, A. R. A theory of Pavlovian conditioning: Variations in the effectiveness of reinforcement and nonreinforcement. In A. Black \& W. F. Prokasy (Eds.), Classical conditioning II, Current theory and research. New York: Appleton-Century-Crofts, 1972.

(Received for publication November 29, 1977; revision accepted November 21, 1978.) 